\title{
The Efficacy of Autologous Nebulized Platelet Rich Plasma (PRP) As an Early Adjuvant Therapeutic and Prognostic Treatment Modality in the Management of Inhalation Lung Injury
}

\author{
SHERIENE METWALLY SALAMA, M.D.; IBRAHIM HUSSEIN KAMEL, M.D.; \\ MOHAMED GHANEM, M.D. and AHMAD F. EL-SHERIF, M.D. \\ The Department of Plastic, Burns and Maxillofacial Surgery, Faculty of Medicine, Ain Shams University, Cairo, Egypt
}

\begin{abstract}
Background: Despite considerable advances in our knowledge of the pathophysiology of inhalation injury, there are few specific therapeutic options, and patient care is mainly supportive. Platelets release growth factors and other immune system components to start healing process. By using nebulized Platelet Rich Plasma (PRP), platelets may reduce inflammation and stimulates bronchial tree healing. However, there are no clear data supporting or refuting the use of inhaled PRP in inhalation injury.
\end{abstract}

Objective: To evaluate the role of nebulized autologous Platelet Rich Plasma (PRP) in management of Smoke Inhalation Injury (SII) as early adjuvant therapeutic and prognostic modality.

Methods: This is prospective study that included 40 patients with burns 25-50\% TBSA and have inhalation lung injury. They were divided into 2 groups; 20 patients received aerosolized particles of nebulized PRP beside regular regimen. The other control group included 20 patients and received regular treatment regimen only.

Results: There was a statistical significant difference $(p<0.01)$ of biologic changes in upper airway with smoke injury in study group patients compared to control group patients who received regular protocol measured at the same follow-up time interval, relatively same TBSA and initial bronchoscopy scoring. The mean extubation time was $7 \pm 1$ days in study group compared to $14 \pm 1$ days in control group. Study group less showed shorter hospital stay and less mortality rate.

Conclusion: The application of aerosolize the PRP solution in the nebulizer with regular treatment protocol of inhalation burn patients could be a beneficial therapeutic tool for regenerating of post burn damaged tracheo-broncho-pulmonary tree. Further studies on larger patient population with clinical and pathological evidences are warranted.

Key Words: Smoke inhalation injury - Respiratory management - PRP inhalation.

\section{INTRODUCTION}

Inhalation injury is defined as an injury to the epithelial lining of tracheobronchial tree and lower airways [1]. Smoke Inhalation Injury (SII) is a major cause of morbidity and mortality in burn patients as it can trigger respiratory failure, pneumonia, septicaemia and ARDS [2]. Enclosed-space fires, loss of consciousness, facial burns and large TBSA $(>40 \%)$ should raise the suspicion of SII. Ideally, all victims suspected of SII should undergo fibreoptic bronchoscopy to evaluate the possibility of SII [3].

Fibreoptic Bronchoscopy (FOB) is the gold standard for the diagnosis and evaluation of the severity of airway inhalation injury. In addition, it is often used for airway hygiene, removing particulate matter, mucus plugging obstructing bronchi, and the large quantity of inflammatory secretion that forms because of cellular necrosis [5]. Classically, imaging tests such as chest X-rays and CT scans have little or no value in the diagnosis of SII [8].

Table (1): Abbreviated injury score grading scale for inhalation injury on bronchoscopy [9].

\begin{tabular}{cll}
\hline Grade & Class & Description \\
\hline 0 & No injury & $\begin{array}{l}\text { - Absence of carbonaceous deposits, ery- } \\
\text { thema, edema, bronchorrhea, or obstruc- } \\
\text { tion. }\end{array}$ \\
1 & $\begin{array}{l}\text { Mild } \\
\text { injury }\end{array}$ & $\begin{array}{l}\text { - Minor or patchy areas of erythema, } \\
\text { carbonaceous deposits, bronchorrhea, } \\
\text { or bronchial obstruction. }\end{array}$ \\
2 & $\begin{array}{l}\text { Moderate } \\
\text { injury }\end{array}$ & $\begin{array}{l}\text { - Moderate degree of erythema, carbon- } \\
\text { aceous deposits, bronchorrhea, or bron- } \\
\text { chial obstruction. }\end{array}$ \\
3 & $\begin{array}{l}\text { Severe } \\
\text { injury }\end{array}$ & $\begin{array}{l}\text { - Severe inflammation with friability, } \\
\text { copious carbonaceous deposits, bronch- } \\
\text { orrhea, or obstruction. }\end{array}$ \\
4 & $\begin{array}{l}\text { Massive } \\
\text { injury }\end{array}$ & $\begin{array}{l}\text { Evidence of mucosal sloughing, necro- } \\
\text { sis, endoluminal obstruction. }\end{array}$ \\
\hline
\end{tabular}


Supportive respiratory care remains essential in managing inhalation injury. Adjuncts still lacking definitive evidence of efficacy include bronchodilators, mucolytic agents, inhaled anticoagulants, nonconventional ventilator modes, prone positioning, and extracorporeal membrane oxygenation. Recent research focusing on molecular mechanisms involved in inhalation injury has increased the number of potential therapies [10].

Platelet-Rich Plasma (PRP) is an innovative and promising approach in tissue regeneration. PRP is defined as an autologous concentrated preparation of platelets and their associated growth factors in a small volume of plasma [11].

Aside from coagulation factors, platelets store and release many bioactive antigenic factors [12] and attract inflammatory cells that enhance angiogenesis, tissue regeneration, and wound healing [13].

Therefore, Platelet-Rich Plasma (PRP), which includes concentrated platelets, has been extensively used to accelerate tissue regeneration in various medical fields. However, the signalling mechanism by which PRP controls angiogenesis and tissue regeneration remains unknown. Recently, it has been reported that soluble extract from mouse PRP stimulates angiogenesis and maintains lung vascular integrity [14].

Recently, PRP is used as a treatment option for lung inflammation via nebulized PRP. This new delivery method increases the number of platelets into the lungs' endothelial cells, which reduces inflammation and stimulates healing process [15].

In this study, patients with smoke inhalation injury were treated with nebulized PRP beside regular supportive regimens aiming to evaluate its role in management of smoke inhalation injury and tissue regeneration.

\section{PATIENTS AND METHODS}

This was a prospective study in Burn Unit of Ain Shams University Hospitals. It was done between March 2015 to March 2017.

This study included 40 burned patients with SII; they were divided into two groups, study group that received nebulized PRP and control group. Inclusion criteria included: Age from 10-60 years, TBSA less than 50\% with suspected SII (based on smoke exposure within a confined space or soot at the nares, pharynx, larynx, facial burns) who require mechanical ventilation within $48 \mathrm{~h}$ of admission. Approval of our institutional Ethics Committees for the study protocol and written informed consent were obtained from each patient's family.

\section{Measurements:}

The clinical data recorded for all cases under study and control group included age, sex, TBSA, associated injuries, chest radiograph, inhalation injury grade by bronchoscopy which was performed according to a standardized protocol within $24 \mathrm{~h}$ of admission, initial arterial blood gas analysis, $\mathrm{PaO}_{2} / \mathrm{FiO}_{2}$. ( $\mathrm{P} / \mathrm{F}$ ratio, the ratio between the arterial partial pressure of oxygen and the fraction of inhaled oxygen is indicative of relative hypoxemia).

The Carboxyhaemoglobin ( $\mathrm{COHb}$ ) level was noted from the ABG measurement taken on admission (only the values were used that were taken less than $2 \mathrm{~h}$ post-injury due to the short half-life of carbon monoxide especially with $100 \%$ oxygen). Follow-up for plasma (CO-Hb) level was done every other day.

Duration of mechanical ventilation, Intensive Care Unit (ICU) stay, pneumonia development, and patient outcome also were documented. Followup measurements include Arterial Blood Gas (ABG) analysis every day and airway inspection by bronchoscopy every week, the degree of bronchial mucosal status was evaluated by using a standardized bronchoscopy scoring system based on the Abbreviated Injury Score (AIS) criteria.

Extubation criteria were adequate when consciousness and capacity to maintain adequate arterial oxygen partial pressure/inspired oxygen fraction ratio $\left(\mathrm{PaO}_{2} / \mathrm{FiO}_{2}=\mathrm{P} / \mathrm{F}\right.$ ratio $)>200$ provided by using simple oxygen devices $\left(\mathrm{FiO}_{2}<0.4\right.$ and with low levels of Positive End-Expiratory Pressure (PEEP) of $<5 \mathrm{~cm} \mathrm{H}_{2} \mathrm{O}$ ). The study defined pneumonia as consolidation on the chest radiograph film, body temperature of $>38^{\circ} \mathrm{C}$ or $\angle 36^{\circ} \mathrm{C}$, white blood cell count of $\geq 12,000$ cells $/ \mathrm{mm}^{3}$ or $\leq 4000$ cells $/ \mathrm{mm}^{3}$, and positive culture of sputum or endotracheal aspirates except for normal respiratory/ oral flora.

The time period (in days) during which mechanical ventilation was required was also recorded.

\section{Methods:}

- Obtain blood sample by venepuncture in Acid Citrate Dextrose (ACD) tubes.

- Do not chill the blood at any time before or during platelet separation.

- Centrifuge the blood using a 'soft' spin (1500rpm, $20^{\circ} \mathrm{C}, 10$ minutes).

- Transfer the supernatant plasma containing platelets into another sterile tube (without anticoagulant). 
- Centrifuge tube at a higher speed (a hard spin) (2000rpm, $20^{\circ} \mathrm{C}, 15$ minutes) to obtain a platelet concentrate.

- The lower $1 / 3^{\text {rd }}$ is PRP and upper $2 / 3^{\text {rd }}$ is Plateletpoor Plasma (PPP). At the bottom of the tube, platelet pellets are formed.

- Remove PPP and suspend the platelet pellets in a minimum quantity of plasma $(2-4 \mathrm{~mL})$ by gently shaking the tube.

Treatment strategy applied for 20 patients as control group was according to an evidence-based protocol for patients with smoke inhalation injury that applied in our Burn Unit [16].

- Titrate humidified high-flow oxygen to maintain $\mathrm{SaO}_{2} \mathrm{~s}>90 \%$.

- Cough, deep breath exercises every 2 hours.

- Turn patient side to side every 2 hours.

- Chest physiotherapy every 2 hours.

- Nebulize $20 \% \mathrm{~N}$-acetylcysteine $(3 \mathrm{ml})$ every 4 hours for 7 days. Co-nebulize with a bronchodilator (albuterol) if wheezing.

- Alternate aerosolizing 5,000-10,000 units of heparin (in $3 \mathrm{ml}$ normal saline) every 4 hours for 7 days.

- Nasotracheal suctioning as needed.

- Early ambulation.

- Sputum cultures for intubated patients every MW-F.

- Pulmonary function studies at discharge and at outpatient visits.

- Patient/family education about the disease process.

Study group, in addition to classical regimen, they received aerosolize ( $3 \mathrm{ml})$ PRP solution in the nebulizer with oxygen every day till extubation criteria were adequate.

\section{Statistical analysis:}

All data was analysed using SPSS (statistical package for the social sciences, Version 12) and displayed as mean \pm SD. The level of significance was $p<0.01$. The pearson correlation coefficient was used to assess the associations between different parameters. Student's $t$-tests were used to investigate differences between study and control group of each parameter. Analysis of the combined effect of gender, age, COHb, TBSA, bronchoscopy findings, and $\mathrm{P} / \mathrm{F}$ ratio was performed using logistic regression. The logistic regression model was tested on the sample of patients with complete data $(n=40)$.

\section{RESULTS}

40 patients (proved by bronchoscopy to have SII) were divided randomly into study and control group with relatively same patient criteria in both groups. TBSA burn was not significantly different (30.7\% in study and $28.8 \%$ in control group). 31 patients survived, and 9 patients died with mortality rate $23 \%$. Mortality was almost twice as great in control group (20\%) than study group (10\%). There was positive correlation between TBSA and mortality $(p<0.01)$. The causes of death were sepsis, multi-organ failure $(n=5)$ and acute respiratory distress syndrome ARDS $(n=4)$. The mean age of death (48.79) was significantly higher than survivors (39.28).

Baseline characteristics and the clinical course of the 40 patients are shown in (Table 2).

Table (2): Demographics and clinical course of the patients.

\begin{tabular}{ll}
\hline Variable & Patients \\
\hline Number of patients & 40 \\
Age (mean, in year) & $37.7(15-60)$ years \\
Female/Male & $15 / 25$ \\
Facial burns (extensive/mild) & $28 / 12$ \\
Diagnostic fiberoptic bronchoscopy & 40 \\
Intubated and ventilated & $7 \pm 1$ days \\
Tracheostomy & non \\
Death ratio study/control & $20 \% / 10 \%$ \\
\hline
\end{tabular}

There was a significant difference in $\mathrm{P} / \mathrm{F}$ ratios $(p<0.01)$ between patients who had received PRP inhalation therapy (mean P/F ratio of 36.24) and those that received regular treatment (mean $\mathrm{P} / \mathrm{F}$ ratio 25.17). Examining the relationship between bronchoscopic findings and $\mathrm{P} / \mathrm{F}$ ratio showed that the $\mathrm{P} / \mathrm{F}$ ratio decreased as the severity of the inhalation injury increased.

The half-life of COHgb when breathing $100 \%$ high-flow oxygen is 20 minutes, therefore, the concentration of carboxyhaemoglobin is reduced to approximately $50 \%$ every 20 minutes [17].

The bronchoscopic scoring of early-discharge patients, whose hospital stays were $<5$ days, was mainly grade 1 and 2 with no significant difference between study and control groups. There was a significant difference $(p<0.01)$ of biologic changes in upper airway with smoke injury (decreased oedema formation, decreased mucus production, decreased tissue inflammation) in study group. The mean extubation time was $7 \pm 1$ days in study group compared to $14 \pm 1$ days. The mean hospital stay was $15 \pm 1$ days in study group to $23 \pm 1$ days in control group Figs. (1A-C). 

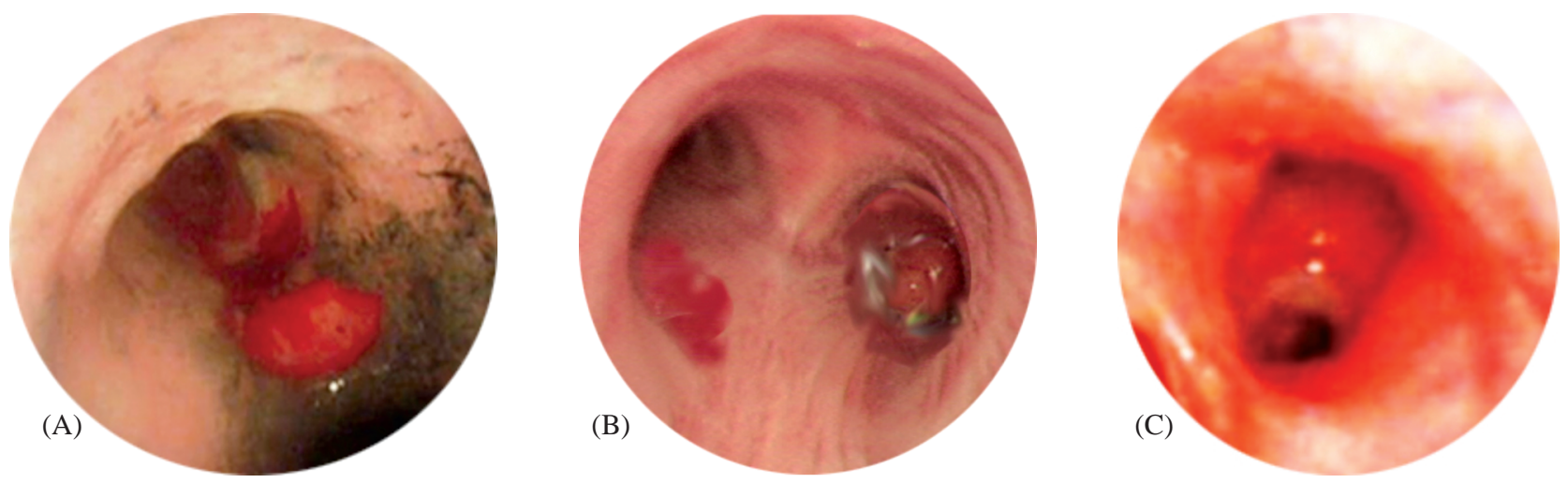

Fig. (1): (A) Bronchoscopy photo of a 22 years old male patient with $25 \%$ TBSA. The photo is taken 18 hrs after inhalation injury. There is evidence of mucosal sloughing, carbonaceous deposits, necrosis \& endoluminal obstruction, (B) Bronchoscopy photo 4 days after PRP treatment showing moderate erythema and carbonaceous deposits. (C) Photo 10 days after PRP treatment showing reduced erythema and absence of carbonaceous deposits.
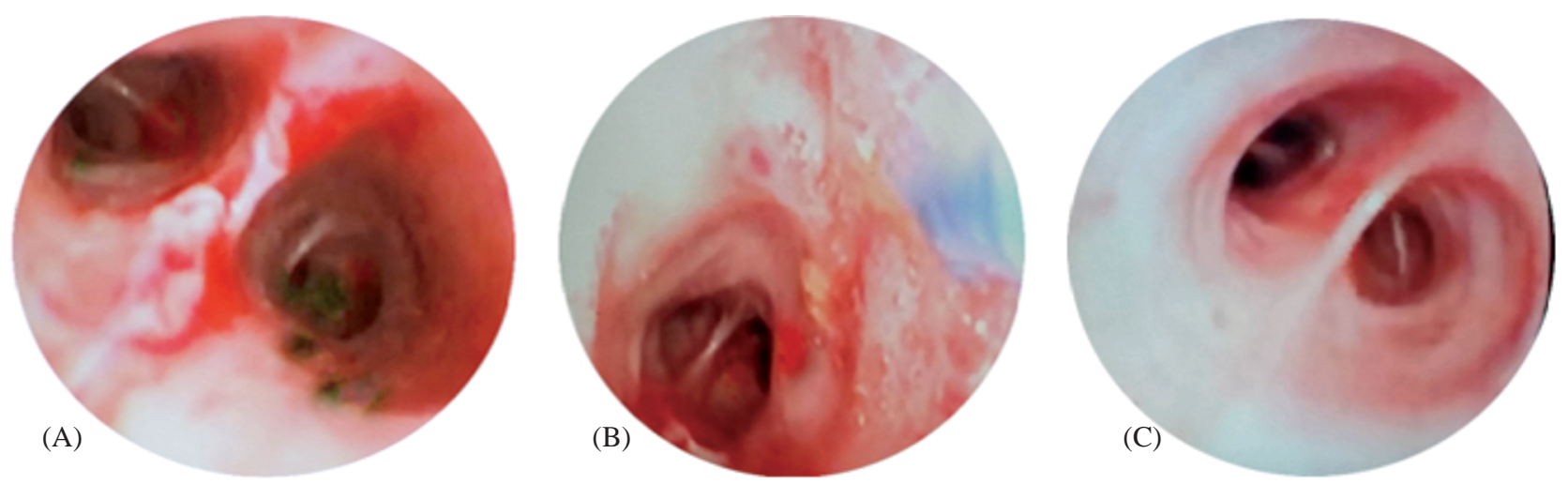

Fig. (2): (A) Bronchoscopy photo of a 31 years old female patient with $20 \%$ TBSA. The photo is taken 24 hrs after inhalation injury. There is evidence of mucosal sloughing, carbonaceous deposits \& necrosis (B) Bronchoscopy Photo 4 days after PRP treatment showing moderate erythema. (C) Photo 10 days after PRP treatment showing reduced erythema and absence of carbonaceous deposits.
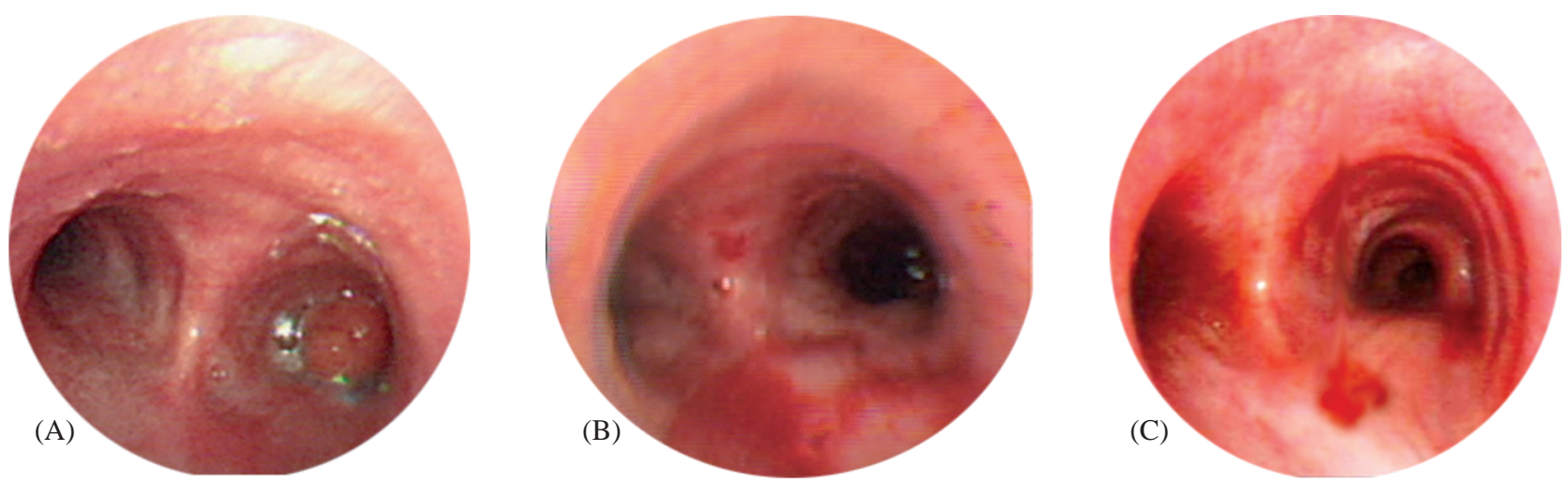

Fig. (3): (A) Bronchoscopy photo of a 35 years old female patient with $21 \%$ TBSA. The photo is taken 24 hrs after inhalation injury. There is evidence of mucosal sloughing, carbonaceous deposits, obstruction \& necrosis (B) Bronchoscopy photo 4 days after regular treatment showing erythema and moderate obstruction. (C) Photo 10 days after regular treatment showing reduced erythema, moderate obstruction and absence of carbonaceous deposits.

\section{DISCUSSION}

A major challenge in smoke inhalation injury research is the accurate diagnosis and grading of injury severity. Successful treatment of smoke inhalation injury will depend not only on accurate diagnosis and grading, but also on the development of therapies that target both airway and parenchymal injuries. Airway management should focus on reducing airway hyperemia/edema, ameliorating or preventing bronchospasm, reducing mucus secretion, preventing/lysing airway fibrin clots, and 
repairing airway epithelium to improve mucociliary clearance. Lung parenchymal management should target increased permeability of both the pulmonary microvascular and alveolar epithelium as well as parenchymal inflammation [18].

Pilot studies of potential treatment strategies are underway including those focused on: Antiinflammatory agents, Nitric Oxide Synthase (NOS) inhibitors, antioxidants, anti-mucus secretion agents, anticoagulants, fibrinolytic agents, specific bronchodilators, neuropeptide modulators, and cyclooxygenase inhibitors. Additionally, cellular therapy currently in the preclinical stage offers the possibility of accelerating airway healing and favourably altering the pathophysiologic changes. Recently, it was found that administration of adipose tissue-derived mesenchymal stem cells either intravenously or through nebulization may hold potential for treating smoke inhalation damage [19].

In our study we tried to evaluate the effectiveness of nebulized autologous Platelet Rich Plasma (PRP) in treatment of acute inhalation injury as a biological adjunct to other methods.

Currently, there is a paucity of critical scientific data regarding the beneficial effects of platelet rich plasma in clinical procedures. There have been animal and human studies both purporting and refuting its adjunctive positive effect. In theory, PRP has many beneficial effects such as autologous supply of growth factors and wound healing. In addition, it is relatively inexpensive and readily available. Recently, it has reported that soluble Platelet-Rich Plasma (PRP) extract, which contains abundant angiopoietin-1 and multiple other angiogenic factors, stimulates angiogenesis and maintains vascular integrity in vitro and in vivo [20].

Our results indicate that by allowing the patient to breathe aerosolized particles of nebulized PRP, the new delivery method increases the number of platelets into the trachea-broncho-pulmonary endothelial cells, which reduces inflammation and stimulates healing process. Inhaled PRP extract restores mucosal integrity as shown by serial repeated endoscopic examination and repeated Bronchoalveolar Lavage (BAL) to remove secretions, slough and carbonaceous material till the airways became clear. After lavage, there was a progressive improvement of all bronchoscopic findings.

There was a significant difference $(p<0.01)$ of biologic changes in upper airway with smoke injury (decreased oedema formation, decreased mucus production, decreased tissue inflammation) in study group patients who inhaled PRP compared to control group measured at the same follow-up time interval, relatively same TBSA and initial bronchoscopic scoring. The mean extubation time was $7 \pm$ 1 days in study group compared to $14 \pm 1$ days. The mean hospital stay was $15 \pm 1$ days in study group compared to $23 \pm 1$ days in control group.

\section{Conclusion:}

Based on outcome data, we propose that the application of aerosolize PRP solution in the nebulizer with oxygen in treatment protocol of inhalation-injured burn patients could be a potentially good therapeutic tool for damaged trachea-bronchopulmonary tree. Further clinical and pathological evidence is needed to substantiate the generalizability of these findings among burned patients with SII.

\section{REFERENCES}

1- W.R. Clark: Smoke inhalation; diagnosis and treatment, World J. Surg., 16: 24, 1992.

2- E.C. Kimmel and K.R. Still: Acute lung injury, acute respiratory distress syndrome and inhalation injury: An overview, Drug Chem. Toxicol., 22: 91, 1999.

3- L.C. Cancio: Airway management and smoke inhalation injury in the burn patient, Clin. Plast. Surg., 36: 555, 2009.

4- H. Yamamura, S. Kaga, K. Kaneda and Y. Mizobata: Chest computed tomography performed on admission helps predict the severity of smoke-inhalation injury, Crit. Care, 17: R95, 2013.

5- R.P. Mlcak, O.E. Suman and D.N. Herndon: Respiratory management of inhalation injury, Burns, 33 (1): 2-13, 2007.

6- M.J. Mosier, T.N. Pham, D.R. Park, J. Simmons, M.B. Klein and N.S. Gibran: Predictive value of bronchoscopy in assessing the severity of inhalation injury, J. Burn Care Res., 33 (1): 65-73, 2012.

7- J.M. Albright, C.S. Davis, M.D. Bird, L. Ramirez, H. Kim, E.L. Burnham, et al.: The acute pulmonary inflammatory response to the graded severity of smoke inhalation injury, Crit. Care Med., 40 (4): 1113-21, 2012.

8- R.P. Mlcak, O.E. Suman and D.N. Herndon: Respiratory management of inhalation injury, Burns, 33 (1): 2-13, 2007.

9- Albright J.M., Davis C.S., Bird M.D., Ramirez L., Kim H., Burnham E.L., et al.: The acute pulmonary inflammatory response to the graded severity of smoke inhalation injury. Crit. Care Med., 40: 1113-21, 2012.

10- Challenges in Research on the Pathophysiology of Smoke Inhalation Injury and Its Clinical Management Lancet. October 01; 388 (10052): 1437-1446. doi:10.1016/S01406736 (16)31458-1, 2016.

11- Platelet-rich plasma: A review of biology and applications in plastic surgery. Eppley B.L., et al.: Plast. Reconstr. Surg., 2006. 
12- Klement G.L., Yip T.T., Cassiola F., Kikuchi L., Cervi D., Podust V., Italiano J.E., Wheatley E., Abou-Slaybi A., Bender E., et al.: Platelets actively sequester angiogenesis regulators. Blood, 113: 2835-2842, 2009.

13- Demidova-Rice T.N., Wolf L., Deckenback J., Hamblin M.R. and Herman I.M.: Human platelet-rich plasma-and extracellular matrix-derived peptides promote impaired cutaneous wound healing in vivo. PLoS One, 7: e32146, 2012.

14- Mammoto T., Jiang A., Jiang E. and Mammoto A.: Platelet rich plasma extract promotes angiogenesis through the angiopoietin1-Tie2 pathway. Microvasc. Res., 89: 15-24, 2013.

15- Nebulizer Applies PRP Directly to Damaged Lungs NEWS PROVIDED BY Dr. Prabhat. Soni. Jul. 13, 08: 40 ET, 2015.

16- Challenges in Research on the Pathophysiology of Smoke Inhalation Injury and Its Clinical Management Lancet.
October 01; 388 (10052): 1437-1446. Doi: 10.1016/S01406736(16)31458-1, 2016.

17- Prockop L. and Chichkova R.: Carbon monoxide intoxication: An updated review. J. Neurol. Sci., 262: 122-30. [PubMed], 2007.

18- Yurt R.W., Bessey P.Q., Bauer G.J., et al.: A regional burn center's response to a disaster: September 11, 2001, and the days beyond. J. Burn Care Rehabil., 26 (2): 117-124. [PubMed: 15756112], 2005.

19- Ihara K., Fujuda S., Enkhtaivan B., et al.: Abstract 19670: Adipose-derived Mesenchymal Stem Cells Attenuate Pulmonary MicrovascularHyperpermeability After Smoke Inhalation. Circulation, 132 (Suppl 3): A19670, 2015.

20- Acceleration of Lung Regeneration by Platelet-Rich Plasma Extract through the Low-Density Lipoprotein Receptor-Related Protein 5-Tie2 Pathway Am. J. Respir. Cell. Mol. Biol., Jan., 54 (1): 103-113, 2016. 